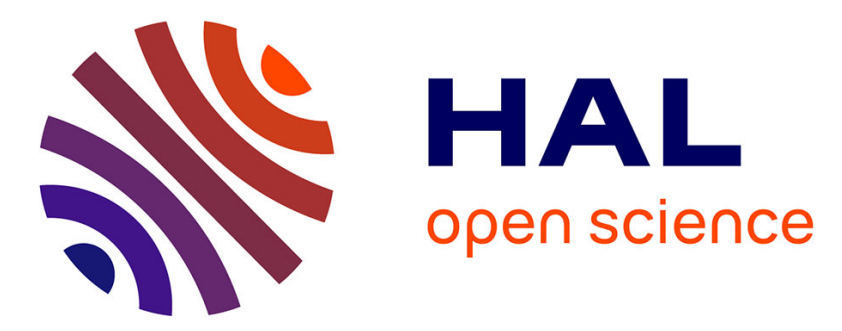

\title{
Surface reconstruction from routine CT-scan shows large anatomical variations of falx cerebri and tentorium cerebelli
}

Hélène Staquet, Pierre-Marc François, Baptiste Sandoz, Sébastien Laporte, Philippe Decq, Stéphane Goutagny

\section{To cite this version:}

Hélène Staquet, Pierre-Marc François, Baptiste Sandoz, Sébastien Laporte, Philippe Decq, et al.. Surface reconstruction from routine CT-scan shows large anatomical variations of falx cerebri and tentorium cerebelli. Acta Neurochirurgica, 2020, 162, pp.1-7. 10.1007/s00701-020-04256-2 . hal03026425

\section{HAL Id: hal-03026425 \\ https://hal.science/hal-03026425}

Submitted on 26 Nov 2020

HAL is a multi-disciplinary open access archive for the deposit and dissemination of scientific research documents, whether they are published or not. The documents may come from teaching and research institutions in France or abroad, or from public or private research centers.
L'archive ouverte pluridisciplinaire HAL, est destinée au dépôt et à la diffusion de documents scientifiques de niveau recherche, publiés ou non, émanant des établissements d'enseignement et de recherche français ou étrangers, des laboratoires publics ou privés. 


\title{
Surface reconstruction from routine CT-scan shows large anatomical variations of falx cerebri and tentorium cerebelli
}

\author{
Hélène Staquet ${ }^{1,2,3} \cdot$ Pierre-Marc Francois ${ }^{2} \cdot$ Baptiste Sandoz $^{2} \cdot$ Sébastien Laporte $^{2} \cdot$ Philippe Decq $^{1,2,3}$. \\ Stéphane Goutagny ${ }^{1,2,3}$ [B]
}

\begin{abstract}
Background Finite element modeling of the human head offers an alternative to experimental methods in understanding the biomechanical response of the head in trauma brain injuries. Falx, tentorium, and their notches are important structures sur-rounding the brain, and data about their anatomical variations are sparse.

Objective To describe and quantify anatomical variations of falx cerebri, tentorium cerebelli, and their notches.

Methods 3D reconstruction of falx and tentorium was performed by points identification on 40 brain CT-scans in a tailored Matlab program. A scatter plot was obtained for each subject, and 8 anatomical landmarks were selected. A reference frame was defined to determine the coordinates of landmarks. Segments and areas were computed. A reproducibility study was done. Results The height of falx was $34.9 \pm 3.9 \mathrm{~mm}$ and its surface area $56.5 \pm 7.7 \mathrm{~cm}^{2}$. The width of tentorium was 99.64 $\pm 4.79 \mathrm{~mm}$ and its surface area $57.6 \pm 5.8 \mathrm{~cm}^{2}$. The mean length, height, and surface area of falx notch were respectively $96.9 \pm 8$ $\mathrm{mm}, 41.8 \pm 5.9 \mathrm{~mm}$, and $28.8 \pm 5.8 \mathrm{~cm}^{2}$ (range $15.8-40.5 \mathrm{~cm}^{2}$ ). The anterior and maximal widths of tentorial notch were 25.5 $\pm 3.5 \mathrm{~mm}$ and $30.9 \pm 2.5 \mathrm{~mm}$; its length $54.9 \pm 5.2 \mathrm{~mm}$ and its surface area $13.26 \pm 1.6 \mathrm{~cm}^{2}$. The length of falx notch correlated with the length of tentorial notch $(r=0.62, P<0.05)$.
\end{abstract}

Conclusion We observe large anatomical variations of falx, tentorium, and notches, crucial to better understand the biomechanics of brain injury, in personalized finite element models.

Keywords Modeling $\cdot$ Brain herniation $\cdot$ Brainstem $\cdot$ Corpus callosum injury $\cdot$ Reference frame

\section{Introduction}

Brain hemispheres and cerebellum are separated by two rigid structures: the falx cerebri and the tentorium cerebelli. Falx and tentorium are stiffer than brain and pia. They are composed of collagen-rich fibrous tissues which are responsible for the high resistance of these structures to tensile strength $[1,25]$.

Stéphane Goutagny

stephane.goutagny@aphp.fr

1 Neurosurgery, Assistance Publique Hôpitaux de Paris, Hôpital Beaujon, 100 boulevard du Général Leclerc, 92110 Clichy, France

2 Arts et Metiers, IBHGC, 151 bd de l'Hopital, 75013 Paris, France

3 Université de Paris, Université Denis Diderot Paris 7, Paris, France
Falx is a midline reflection of dura mater, sickle-shaped, that extends vertically between the two cerebral hemispheres. The free margin of the falx shapes its inferior limit and borders the corpus callosum. The superior sagittal sinus, which is attached to the skull, limits the outer margin. The tentorium, crescent-shaped, extends between the cerebellum and the cerebral hemispheres. The tentorium forms the upper limit of the posterior fossa. Its aperture, called the tentorial notch, is the communication between supratentorial and infratentorial spaces. The tentorial notch contains one important structure, the brainstem, surrounded by cerebrospinal fluid. The shape of the tentorial is triangular, with two free edges, joining at the tentorial apex. The dorsum sellae forms its anterior edge. The outer margin of tentorium is outlined by the straight sinus on the top, the transverse sinus posteriorly, and the superior petrosal sinus anteriorly [21].

Because of their shape, stiffness, and location within the cranium, these structures affect the transfer of mechanical loads within the brain, constraining brain motion, and 
dampening deformation across the midline [3]. Herniation occurs when e.g. a hematoma or a tumor induces brain shift across these notches. Subfalcine and trantentorial herniations can lead to severe disability or death. Hardy et al. examined on using human cadavers the brain displacement and deformation during impact. Dynamic impact events in the vicinity of the falx cerebri influence the brain motion patterns more drastically for impacts in the coronal and horizontal planes rather than in sagittal plan [9]. Using finite element model of the head, Ho et al. studied the strain distribution in the brain and the biomechanical influence of the falx and tentorium during impacts. They demonstrated that falx and tentorium could have an effect on the biomechanical behavior of surrounding brain tissues, especially corpus callosum and brainstem [11]. Thus, the variations of the size of falx and tentorium could modify strain distribution within the cranium.

Little attention has been given to anatomical variations of falx and tentorium. Only one study reporting the variations in the height of the falx had been done so far on living subjects, and was based on carotid angiography in 1969 [8]. Few studies address the anatomy of the tentorial notch. These studies were performed on cadaveric cases [2, 5, 19, 22]. There is a lack of data on anatomical variations and correlations on both tentorium and falx in living subjects.

Anatomical variations of falx, tentorium, and their notches could influence outcomes of brain herniations. Thus, the aim of this study is to characterize anatomical variations of the falx and the tentorium, focusing on their notches. We propose a new method to measure falx and tentorium using routine $\mathrm{CT}$ imaging on adult subjects.

\section{Patients and method}

Forty anonymized brain CT scans were extracted from institutional database (AP-HP, Approval no. 2017-013 from the CNIL, authorization no. 1980120). CT scans were randomly selected excluding cases with midline deviation. 3D computed-tomography (CT) scans were routinely acquired on a REVOLUTION DISCOVERY $750 \mathrm{HD}$ or DISCOVERY CT750 (General Electric System, Boston, USA) with voxel resolutions ranging from $0.416 \times 0.416 \times$ 1.250 to $0.576 \times 0.576 \times 1.250 \mathrm{~mm}$.

Anonymous DICOM images were implemented in a homemade Matlab program (MathWorks ${ }^{\circledR}$ version 7.0, available on request). One senior surgeon (HS) thoroughly pointed each anatomical structure (falx, tentorium, and venous sinuses) on axial CT slices. Points were identified every c.a $5 \mathrm{~mm}$, one slice out of two. Per subject, on average 1386 points were identified (650 for the falx, 440 for the tentorium and 296 for sinuses). The mean acquisition time was about $20 \mathrm{~min}$ per case. Workflow is described on Fig. 1. Briefly, a scatter plot was obtained in Matlab. Height anatomical landmarks were identified on this 3D scatter plot: anterior (A) and posterior (B) crista galli, left (C) and right (D) anterior clinoid process, left $(\mathrm{E})$ and right $(\mathrm{F})$ parieto-mastoid point, the tentorial apex $(\mathrm{G})$, and the torcular $(\mathrm{H})($ Fig. 1c).

\section{Reference frame of the head}

In order to determine the coordinates of the anatomical landmarks for further computing, we defined a reference frame (Fig. 1c). The origin of the frame was defined as the midpoint of the segment between tentorial apex and torcular [GH]. The normal of the least-square falx cerebri plan defined the $Y$-axis. The cross-product of the $Y$-axis by the vector (posterior crista galli-tentorial apex) defined the $Z$-axis. The $X$-axis is the result of the cross-product of the $Y$-axis by the $Z$-axis. The three axis were normalized in order to get an orthonormal frame where each axis is a unit vector (a vector of length one), orthogonal to each other. The following measures have been obtained from the coordinates of the anatomical landmarks and the projection of the set of the points of the tentorium and the falx. The computation time was about $10 \mathrm{~min}$ per case.

\section{Falx cerebri}

To characterize falx cerebri, we used the 2 parameters depicted Fig. 2. The height of the falx (1) is the distance between the tentorial apex and the superior edge of the falx cerebri along a straight line passing through the midpoint of the clinoid process and the tentorial apex [8]. The surface of the falx $(\mathrm{sF})$ was calculated by a triangulation method, from all the points of the falx projected on the least square falx plan. The notch of the falx was the area limited by the free edge of the falx and the segment between posterior crista galli and tentorial apex [BG]. In order to characterize the notch, we used the 3 parameters depicted Fig. 2. The height of the falx notch (2) is the maximal distance between the free edge of the falx and the segment [BG]. The length of the falx notch (3) is the maximal distance between two points of the free edge of the falx. The surface of the falx notch $(\mathrm{sFN})$ was assessed by a triangulation method. Ratio between surfaces was calculated.

\section{Tentorium cerebelli}

In order to characterize tentorium cerebelli, we used the 2 parameters depicted Fig. 3. The width of the tentorium (4) is the distance between the 2 parietomastoid points [EF]. The surface of the tentorium (sT) is assessed from all the points projected in the frame, and linked by a triangulation method to obtain a surface. To characterize the tentorial notch, we used 4 parameters. The anterior width (5) is the distance between the 2 anterior clinoid processes [CD]. The maximal width (6) is the maximal distance between two points of the tentorial notch's free edges. The length of the tentorial notch (7) is the distance between tentorial apex 


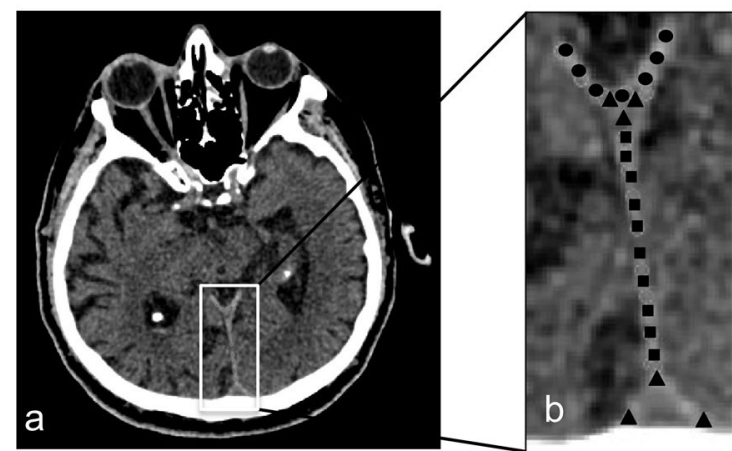

Fig. 1 Pointing acquisition from CT scan. (a) Example of an axial CT scan used. (b) The falx (squares), the tentorium (points), and venous sinuses (triangles) were specified on axial CT. (c) Computing the points allowed to draw a scatter plot. The anatomical landmarks were identified. Anterior crista galli (A): most anterior and inferior point of the falx. Posterior crista galli (B): most posterior and inferior point of the falx. Left anterior clinoid process $(\mathrm{C})$ : most superior and lateral point of the

(G) and the middle point of the 2 clinoid processes segment $[\mathrm{CD}]$. The surface of the tentorial notch (sTN) is calculated by a triangulation method and is delineated by the 2 free edges, the segment $[\mathrm{CD}]$ and the tentorial apex $(\mathrm{G})$. The tentorial angle (8)

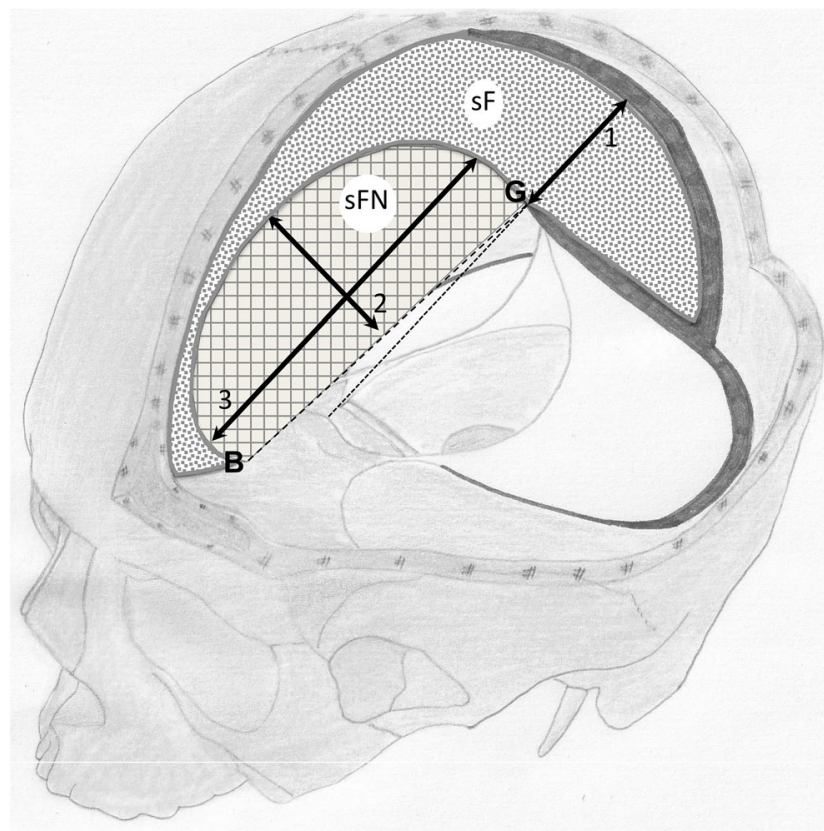

Fig. 2 Parameters describing the falx cerebri. Two anatomical landmarks were first determined: posterior crista galli (B) and tentorial apex $(\mathrm{G})$. Three distances were calculated: the height of the falx (1), the height (2), and the length (3) of the falx notch. Two surfaces were calculated by a triangulation method from projected points: the surface of the falx $(\mathrm{sF})$ and the falx notch $(\mathrm{sFN})$

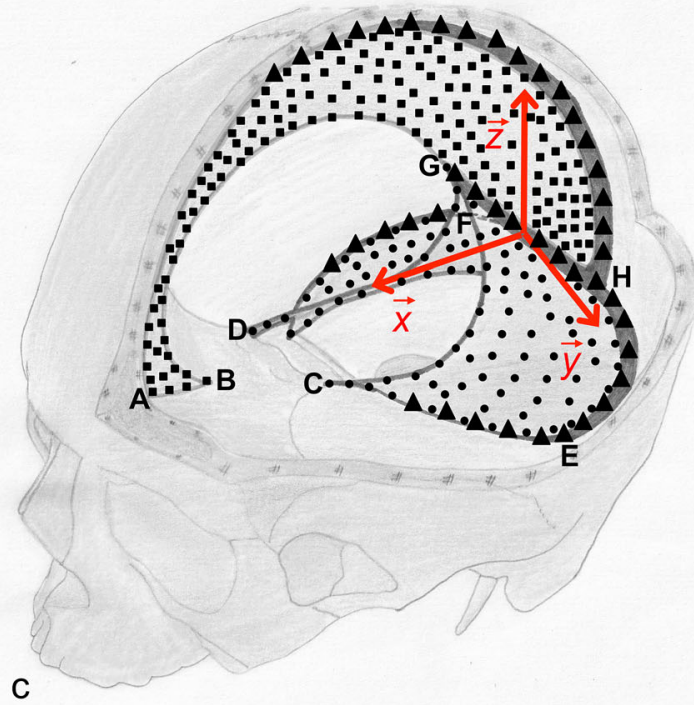

left anterior processus clinoid. Right anterior clinoid process (D): most superior and lateral point of the right anterior processus clinoid. Left parietomastoid point $(\mathrm{E})$ : most lateral point of the tentorium on the left side. Right parietomastoid point $(\mathrm{F})$ : most lateral point of the tentorium on the right side. Tentorial apex $(\mathrm{G})$ : most upper point of the tentorium. Torcular $(\mathrm{H})$ : most posterior and inferior point of the tentorium.

is calculated using the Twining's line, drawn from the midpoint of the segment $[\mathrm{CD}]$ to the torcular $(\mathrm{H})$, and the straight sinus pictured by the segment [GH] [12]. Similarly to the falx, ratio between surfaces was calculated.

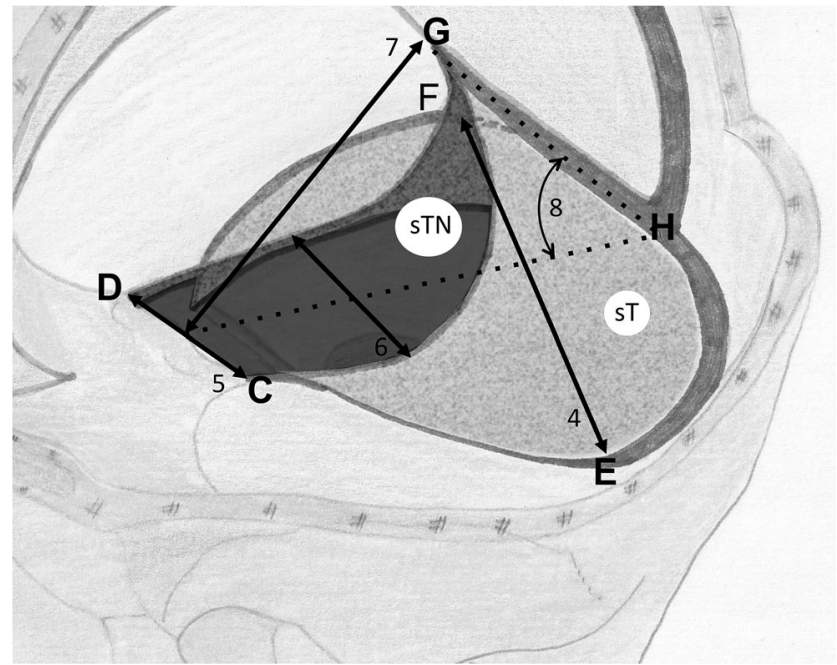

Fig. 3 Parameters describing the tentorium cerebelli and its notch. Six anatomical landmarks were used: left anterior clinoid process (C), right anterior clinoid process (D), left parietomastoid point (E), right parietomastoid point $(\mathrm{F})$, tentorial apex $(\mathrm{G})$, and torcular $(\mathrm{H})$. Four distances were calculated: the width of the tentorium (4), the anterior width (5), the maximal width (6), and the length (7) of the tentorial notch. Surfaces were calculated by a triangulation method from projected points for tentorium (sT) and tentorial notch (sTN). The tentorial angle (8) was also recorded 


\section{Reproducibility study}

To evaluate measurement errors, we carried out a reproducibility study on 6 randomly selected CT-scans. Pointing acquisition and determination of the coordinates of anatomical landmarks had been repeated 3 times for each scan, by the same neurosurgeon, with a minimum $24 \mathrm{~h}$-interval between measures. A non-parametric Spearman test was applied. A measure was considered robust enough when variations were $2 \mathrm{DS}<3 \mathrm{~mm}$ for coordinates, $<5 \%$ for distances and surfaces, and $<5^{\circ}$ for angles.

\section{Statistical analysis}

Results were analyzed using the statistical software XLSTAT 2018.3 (Addinsoft SARL, Bordeaux, FR). All data are presented as mean and standard deviation. The correlation study used a Pearson test, $r>$ or $=0.6$ was considered suitable, with a $P$ value $<0.05$.

\section{Results}

\section{Reproducibility}

The reproducibility error on the coordinates of the anatomical landmarks was less than $1 \mathrm{~mm}$. Two parameters had a reproducibility error superior to $5 \%$ : the anterior width $(5.22 \%)$ and the surface of the tentorial notch $(5.78 \%)$. For the angle, the reproducibility error was less than $2^{\circ}$. These results suggest that the method is reliable and allows further analyses, using our reference frame for the head.

\section{Falx cerebri}

Both distances and surfaces presented large inter-subject variations; e.g., the surface of the falx notch ranged from 15 to $40 \mathrm{~cm}^{2}$ (ratio 2.7) and the height of the falx ranged from 29 to $48 \mathrm{~mm}$ (ratio1.6).

Results are given in Table 1. The falx notch surface represented $33 \% \pm 5.2$ of the whole surface (falx plus falx notch).
As expected, the height of the falx correlates with the surface of the falx $(r=0.6, P<0.05)$. The surface of the falx notch is statistically correlated with its height $(r=0.82, P<0.05)$ but not with its length $(r=0.44, P<0.05)$.

\section{Tentorium cerebelli}

Similarly to falx measurements, distances, angle and surfaces presented large inter-subject variations. Results are given in Table 2. The tentorial notch surface represented $18.79 \% \pm 2.3$ of the whole surface (tentorium plus tentorial notch). The width of the tentorium was not correlated with its surface $(r=0.46$, $P<0.05)$. The surface of the tentorial notch was correlated with its length $(r=0.655, P<0.5)$, but not with its maximal width $(r=0.420, P<0.05)$. We found no correlation between the surfaces of tentorium cerebelli and tentorial notch $(r=0.0, P=$ $0.99)$.

Finally, the length of the falx notch was correlated with the length of the tentorial notch $(r=0.62, P<0.05)$. Surfaces of both notches were not correlated $(r=0.234, P=0.055)$, neither their heights $(r=0.34, P=0.03)$.

\section{Discussion}

\section{Anatomical variations}

The present study presents a consistent description (surfaces and linear measurements) for both falx, tentorium, and their notches, using clinical CT-Scan, on living subjects. Our main results are as follows: the mean surface of the falx was $56.5 \pm 7.7 \mathrm{~cm}^{2}$, and its height $34.9 \pm 3.9 \mathrm{~mm}$. The falx notch has been measured for the first time $\left(28.8 \pm 5.9 \mathrm{~cm}^{2}\right)$. The surface of the tentorium $(57.6 \pm$ $5.8 \mathrm{~cm}^{2}$ ) and the ratio of its free area $(18 \%)$ have been measured for the first time. Important inter-individual variations have been demonstrated in this study for these 2 important structures of the brain. So far the falx and the falx notch have not attracted much attention in the literature. In this study, the height and the surface of the falx notch were the most variable parameters among our data, ranging respectively from 27 to $57 \mathrm{~mm}$ and from 15 to $40 \mathrm{~cm}^{2}$. We found only one study in the literature, in 1969 . It

Table 1 Falx cerebri parameters

\begin{tabular}{|c|c|c|c|c|c|c|}
\hline & \multicolumn{2}{|l|}{ Falx } & \multicolumn{3}{|l|}{ Falx notch } & \multirow[t]{2}{*}{ Surface ratio } \\
\hline & Height (mm) & Surface $\left(\mathrm{cm}^{2}\right)$ & Width (mm) & Height (mm) & Surface $\left(\mathrm{cm}^{2}\right)$ & \\
\hline Minimum & 29 & 43.2 & 81.4 & 27.73 & 15.8 & 24.0 \\
\hline Maximum & 48 & 74.2 & 113.5 & 57.72 & 40.5 & 45.6 \\
\hline 1st quartile & 32.1 & 50.7 & 91.5 & 38.42 & 25.1 & 29.9 \\
\hline Median & 33.8 & 56.8 & 97.1 & 40.45 & 27.3 & 33.1 \\
\hline 3rd quartile & 36.7 & 59.6 & 102 & 144.41 & 32.1 & 37.6 \\
\hline Mean \pm SD & $34.9 \pm 3.9$ & $56.5 \pm 7.7$ & $96.9 \pm 8$ & $41.81 \pm 5.9$ & $28.8 \pm 5.9$ & $33.7 \pm 5.2$ \\
\hline
\end{tabular}


Table 2 Tentorium cerebelli parameters

\begin{tabular}{|c|c|c|c|c|c|c|c|c|}
\hline & \multicolumn{2}{|l|}{ Tentorium } & \multicolumn{4}{|l|}{ Tentorial notch } & \multirow[t]{2}{*}{ Tentorial angle $\left(^{\circ}\right)$} & \multirow[t]{2}{*}{ Surface ratio } \\
\hline & $\begin{array}{l}\text { Width } \\
\text { (mm) }\end{array}$ & $\begin{array}{l}\text { Surface } \\
\left(\mathrm{cm}^{2}\right)\end{array}$ & $\begin{array}{l}\text { Anterior width } \\
(\mathrm{mm})\end{array}$ & $\begin{array}{l}\text { Width } \\
(\mathrm{mm})\end{array}$ & $\begin{array}{l}\text { Length } \\
(\mathrm{mm})\end{array}$ & $\begin{array}{l}\text { Surface } \\
\left(\mathrm{cm}^{2}\right)\end{array}$ & & \\
\hline Minimum & 87.9 & 45.0 & 18.0 & 27.4 & 45.3 & 10.6 & 44.0 & 13.6 \\
\hline Maximum & 111.6 & 70.1 & 36.6 & 38.2 & 66.7 & 16.5 & 66.0 & 24.2 \\
\hline 1st quartile & 97.1 & 54.4 & 23.2 & 29.0 & 50.9 & 11.9 & 50.6 & 17.1 \\
\hline Median & 99.0 & 56.9 & 25.5 & 30.4 & 53.8 & 13.0 & 53.5 & 18.3 \\
\hline 3rd quartile & 103.0 & 62.0 & 27.4 & 32.1 & 58.2 & 14.3 & 57.3 & 20.6 \\
\hline Mean \pm SD & $99.6 \pm 4.8$ & $57.6 \pm 5.9$ & $25.5 \pm 3.5$ & $31.0 \pm 2.5$ & $55.0 \pm 5.3$ & $13.3 \pm 1.7$ & $54.0 \pm 5.0$ & $18.7 \pm 2.3$ \\
\hline
\end{tabular}

was based on carotid arteriography and brought into evidence anatomical variations in the size of the falx, independently of skull dimensions [8]. In this study, the mean height of the falx was $5.27 \mathrm{~cm}$ (ranging from 4 to $6.2 \mathrm{~cm}$ ). The authors studied the interspace between the falx and the corpus callosum (represented by the inferior longitudinal sinus) and its relation to the displacement of the anterior cerebral artery in frontal lesions. It appeared that a narrow falx notch limited the anterior cerebral artery shift. In the present study, the height of the falx was smaller $(3.49 \mathrm{~cm})$. Indeed, we did not include the superior sagittal sinus and straight sinus in the definition of the falx. This could account for the difference. Brockman et al. measured the variations of the superior sagittal sinus (SSS) on CT-scans [4]. The maximal vertical diameter of the SSS, measured $3 \mathrm{~cm}$ dorsally from the coronal suture, was $6.4 \mathrm{~mm}(\mathrm{SD}=1.2)$.

The tentorial notch has been more thoroughly studied because of the clinical importance regarding transtentorial herniation. Transtentorial herniation occurs in a wide variety of neurological conditions. If left untreated, transtentorial herniation progresses rapidly to severe disability or death [7, 17]. Previous studies on tentorium anatomy were made on cadavers, using photographs taken from the superior and lateral views [2, 5, 19, 22]. In 1958, Corsellis et al. described unexpected variations in the size and shape of the tentorial notch [5]. The same year, Sunderland described the relations between this free area and the brainstem, nerves, and vessels [22]. In 1984, using upper view pictures on cadavers, Ono et al. described a maximal tentorium width of $110.8 \mathrm{~mm}$, seemingly different from our results $(99.6 \pm 4.6 \mathrm{~mm})$ [19]. Nevertheless, on the basis that lateral and posterior tentorium margins are firmly attached to the inner surface of the skull, Ono et al. measured the maximum width of the tentorium as the distance between internal bone surfaces. In our study, the lateral sinuses were not included in the definition of tentorium. This could explain the difference in measures. In 2002, Adler and Milhorat analyzed 100 human cadavers, taking upper view pictures during autopsy. He developed a classification system for the tentorial notch, taking into account the length and the width of the notch [2].
Tentorial angle is one of several factors that determine whether the infratentorial approach for surgeries of the pineal or cerebellar vermian region is usable or challenging. One considers to use the supracerebellar infratentorial approach whether the tentorial angle is shallow, or the occipital transtentorial approach whether the tentorial angle is seep [16]. Similarly, the tentorial angle is used to plan the paramedian supracerebellartranstentorial approach for selective amygdalohippocampectomy [23]. A recent trend has emerged to use the Twining line as the reference line to measure the slope of the tentorium. At first, Twining's line, joining the tuberculum sellae and the torcular, on pneumoencephalography, was used to be the landmark of the fourth ventricle [12]. The most recent studies cited above, using MRI, focused only on the tentorial angle using images acquired in the sagittal plane. In two recent studies using the Twining's line, mean tentorial angle was calculated at $42^{\circ}$ and $43.5^{\circ}$, ranging from $25^{\circ}$ to $56.5^{\circ}$, in line with our data $\left(54 \pm 5^{\circ}\right)$ $[16,23]$. Part of the wide range of results could be explained by the precise position of anatomical landmarks used to draw the lines and calculate the angle. The tuberculum sellae is lined up with a line passing through the two anterior clinoid processes and is rather easy to identify in a reproducible way. However, the torcular and the apex are venous confluents with a rather large drop/triangular shape. Depending on the point selected to stand for these landmarks, the calculation of the angle may significantly differ. In this present study, the anatomical landmark called "torcular" is defined as the most posterior and inferior point of the tentorium in the torcular region. The landmark called "apex tentorial" is defined as the most upper point of the tentorium. The exact positions of these landmarks have not been otherwise specified in previous studies [16, 23].

\section{Reference frame}

For 3D modeling of the head, a precise reference frame is mandatory. In this study, we propose a reference frame of the head, as we found no previous publication or recommendations of the International Society of Biomechanics about it. 
This reference frame allows 3D calculations and modeling that was otherwise not possible with pictures on cadavers.

Hernandez et al. used finite element modeling of the brain to investigate the relationships between head motion, falx displacement, and potential damages to corpus callosum [10]. They demonstrated that high coronal and/ or horizontal rotations produce deep lateral displacement of the falx cerebri, which may strain and damage the corpus callosum. They modeled heads with and without falx, and showed that strains in the corpus callosum halved beside when the falx is present. Our study shows important variations of the falx and its notch. One can hypothesize that a large falx notch might behave as an absent falx, thus decreasing damages to the corpus callosum. Pathophysiology of traumatic brain injury is often studied using average finite element model of the head $[11,13,20,24]$. Inter-individual variability of structures, such as the falx and tentorium notches, is seldom taken into account. Our data suggest that including individual anatomical variations of falx and tentorium notches in personalized models could be crucial to better elicit the mechanisms of brain damages.

In our reproducibility study, two parameters had a reproducibility error superior to $5 \%$ (anterior width and surface of the tentorial notch). This variation flows from pixel resolution of routine $\mathrm{CT}$ scans $($ mean $=0.471 \times 0.471 \mathrm{~mm})$. For example, $2 \mathrm{SD}$ for the anterior width of the tentorial notch represent an error of $5.22 \mathrm{~mm}$. This represents an error or 5 pixels for each anatomical landmark. Anatomical landmarks used to assess this measure (clinoid process and torcular) have a volume greater than on pixel [6]. This could also explain the lack of reproducibility for the surface of tentorial notch computed from 3D structures.

\section{Correlation}

We seek after correlations between the 12 anatomical parameters. We found a significant correlation between the length of falx notch and the length of the tentorial notch $(r=0.62$, $P<0.05)$. No data exist in the literature on correlations between these lengths. These 2 structures both originate from the neural crest, but do not appear at the same time during the embryological development. Falx is first discernible in embryos at the age of 41 days, and tentorium is discernible at 51 days $[15,18]$. In addition, some authors studying embryos and adult subjects demonstrated that falx and tentorium follow a different growth pattern. The falx has an anteroposterior growth pattern from the frontal part to the occipital part of the cranium, and the tentorium has a centrifugal growth pattern, from both side of the brainstem in the cerebrocerebellar fissure to a fusion in the median plane posteriorly, along the straight sinus $[14,18]$.

\section{Conclusion}

By precisely defining anatomical landmarks, we showed large variations of the size of falx and tentorium. Because important nervous structures are in close contact with falx and tentorium, their anatomic variations are crucial to better understand the mechanisms of brain herniations. We also propose a reference frame for biomechanical studies of the head.

\section{Compliance with ethical standards}

Conflict of interest The authors declare that they have no conflict of interest.

Ethical approval All procedures performed in studies involving human participants were in accordance with the ethical standards of the institutional and/or national research committee (APHP/CNIL) and with the 1964 Helsinki declaration and its later amendments or comparable ethical standards. For this type of study formal consent is not required. Informed consent was obtained from all individual participants included in the study.

This article does not contain any studies with human participants performed by any of the authors.

\section{References}

1. Adeeb N, Mortazavi MM, Tubbs RS, Cohen-Gadol AA (2012) The cranial dura mater: a review of its history, embryology, and anatomy. Childs Nerv Syst 28(6):827-837

2. Adler DE, Milhorat TH (2002) The tentorial notch: anatomical variation, morphometric analysis, and classification in 100 human autopsy cases. J Neurosurg 96(6):1103-1112

3. Bradshaw DR, Ivarsson J, Morfey CL, Viano DC (2001) Simulation of acute subdural hematoma and diffuse axonal injury in coronal head impact. J Biomech 34(1):85-94

4. Brockmann C, Kunze SC, Schmiedek P, Groden C, Scharf J (2012) Variations of the superior sagittal sinus and bridging veins in human dissections and computed tomography venography. Clin Imaging 36(2):85-89

5. Corsellis JA (1958) Individual variation in the size of the tentorial opening. J Neurol Neurosurg Psychiatry 21(4):279-283

6. Dagtekin A, Avci E, Uzmansel D, Kurtoglu Z, Kara E, Uluc K, Akture E, Baskaya MK (2014) Microsurgical anatomy and variations of the anterior clinoid process. Turk Neurosurg 24(4):484-493

7. Fisher CM (1995) Brain herniation: a revision of classical concepts. Can J Neurol Sci J Can Sci Neurol 22(02):83-91

8. Galligioni F, Bernardi R, Mingrino S (1969) Anatomic variation of the height of the falx cerebri. Its relationship to displacement of the anterior cerebral artery in frontal space-occupying lesions. Am J Roentgenol Radium Therapy, Nucl Med 106(2):273-278

9. Hardy WN, Mason MJ, Foster CD et al (2007) A study of the response of the human cadaver head to impact. Stapp Car Crash J $51: 17-80$

10. Hernandez F, Giordano C, Goubran M, Parivash S, Grant G, Zeineh M, Camarillo D (2019) Lateral impacts correlate with falx cerebri displacement and corpus callosum trauma in sports-related concussions. Biomech Model Mechanobiol 18(3):631-649

11. Ho J, Zhou Z, Li X, Kleiven S (2017) The peculiar properties of the falx and tentorium in brain injury biomechanics. J Biomech 60: 243-247 
12. Isherwood I (1995) Edward wing twining, 1887-1939. AJNR Am J Neuroradiol 16(10):2077-2080

13. Kleiven S, Hardy WN (2002) Correlation of an FE model of the human head with local brain motion-consequences for injury prediction. Stapp Car Crash J 46:123-144

14. Klintworth GK (1967) The ontogeny and growth of the human tentorium cerebelli. Anat Rec 158(4):433-441

15. Klintworth GK (1968) The comparative anatomy and phylogeny of the tentorium cerebelli. Anat Rec 160(3):635-642

16. Lafazanos S, Türe U, Harput MV, Gonzalez Lopez P, Firat Z, Türe H, Dimitriou T, Yaşargil MG (2015) Evaluating the importance of the tentorial angle in the paramedian supracerebellar-transtentorial approach for selective amygdalohippocampectomy. World Neurosurg 83(5):836-841

17. Meyer A (1920) Herniation of the brain. Arch Neurol Psychiatr 4

18. O'Rahilly R, Müller F (1986) The meninges in human development. J Neuropathol Exp Neurol 45(5):588-608

19. Ono M, Ono M, Rhoton AL Jr, Barry M (1984) Microsurgical anatomy of the region of the tentorial incisura. J Neurosurg 60(2): 365-399
20. Raul J-S, Deck C, Willinger R, Ludes B (2008) Finite-element models of the human head and their applications in forensic practice. Int J Legal Med 122(5):359-366

21. Rhoton AL (2000) Tentorial incisura. Neurosurgery 47(3 Suppl): S131-S153

22. Sunderland S (1958) The tentorial notch and complications produced by herniations of the brain through that aperture. Br J Surg 45(193):422-438

23. Syed HR, Jean WC (2018) A novel method to measure the tentorial angle and the implications on surgeries of the pineal region. World Neurosurg 111:e213-e220

24. Tse KM, Lim SP, Tan VBC, Lee HP (2014) A review of head injury and finite element head models. Am J Eng Technol Soc 1(5):28-52

25. Van Noort R, Black MM, Martin TR, Meanley S (1981) A study of the uniaxial mechanical properties of human dura mater preserved in glycerol. Biomaterials 2(1):41-45 